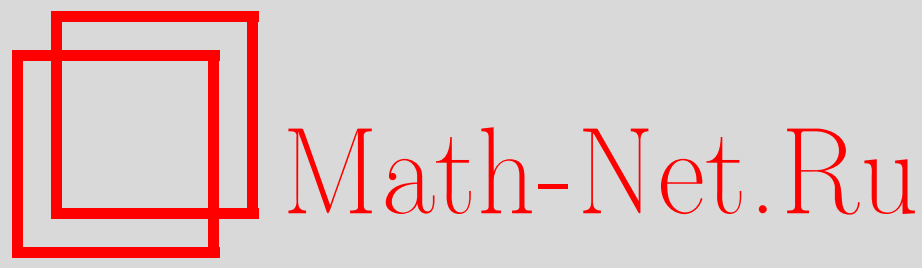

М. О. Корпусов, О разрушении за конечное время решения начально-краевой задачи для нелинейного уравнения ионно-звуковых волн, ТМФ, 2016, том 187, номep 3, 447-454

DOI: https://doi.org/10.4213/tmf8954

Использование Общероссийского математического портала Math-Net.Ru подразумевает, что вы прочитали и согласны с пользовательским соглашением http: //www . mathnet.ru/rus/agreement

Параметры загрузки:

IP : 54.237 .206 .68

26 апреля 2023 г., $16: 25: 43$

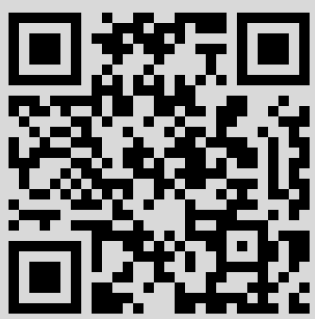




\title{
ФИЗИКА
}

Том 187, № 3

июнь, 2016

(C) 2016 г.

М. О. Корпусов*

\section{О РАЗРУШЕНИИ ЗА КОНЕЧНОЕ ВРЕМЯ РЕШЕНИЯ НАЧАЛЬНО-КРАЕВОЙ ЗАДАЧИ ДЛЯ НЕЛИНЕЙНОГО УРАВНЕНИЯ ИОННО-ЗВУКОВЫХ ВОЛН}

\begin{abstract}
Получены условия разрушения решения начально-краевых задач для одного нелинейного уравнения ионно-звуковых волн в водородной плазме в приближении "горячих" электронов и "тяжелых" ионов. При этом особенностью рассматриваемого нелинейного уравнения является наличие некоэрцитивной нелинейности вида $\partial_{t}|\nabla u|^{2}$, что осложняет его исследование каким-либо энергетическим методом. Представлено решение данной задачи методом нелинейной емкости Похожаева-Митидиери.
\end{abstract}

Ключевые слова: нелинейная емкость, разрушение.

DOI: $10.4213 / \operatorname{tmf} 8954$

\section{1. ВВЕДЕНИЕ}

В работе [1] рассмотрена следующая нелинейная система уравнений динамики "холодных" ионов в приближении самосогласованного поля “горячих" электронов:

$$
\begin{gathered}
\partial_{t} n+\nabla(n \mathbf{v})=0, \\
\partial_{t} \mathbf{v}+(\mathbf{v} \cdot \nabla) \mathbf{v}+\nabla \phi=0, \\
\nabla^{2} \phi=e^{\phi}-n,
\end{gathered}
$$

где $n$ - плотность ионов, $\mathbf{v}$ - скорость ионов, $\phi$ - скалярный потенциал квазистационарного электрического поля, причем $e^{\phi}-$ плотность распределения "горячих" электронов (распределение Больцмана). Уравнение (1.1) - это уравнение непрерывности, уравнение (1.2) - закон сохранения импульса, а (1.3) - уравнение Пуассона.

В приближении безвихревой скорости можно ввести потенциал $\psi$ по формуле $\mathbf{v}=\nabla \psi$. В работе [1] выведено уравнение

$$
\psi_{t t}-\Delta^{2} \psi+\left(|\nabla \psi|^{2}\right)_{t}=0, \quad \Delta^{2} \equiv \Delta \Delta,
$$

* Физический факультет, Московский государственный университет им. М. В. Ломоносова, Москва, Россия. E-mail: korpusov@gmail.com 
где

$$
\Delta \equiv \frac{\partial^{2}}{\partial x_{1}^{2}}+\cdots+\frac{\partial^{2}}{\partial x_{N}^{2}}, \quad N \in \mathbb{N} .
$$

В настоящей работе мы исследуем начально-краевую задачу для уравнения (1.4), которую для удобства перепишем в следующем виде:

$$
\begin{gathered}
u_{t t}-\Delta^{2} u+\left(|\nabla u|^{2}\right)_{t}=0, \quad u(x, 0)=u_{0}(x), \quad u^{\prime}(x, 0)=u_{1}(x), \\
\left.u\right|_{\partial \Omega}=\left.\frac{\partial \Delta u}{\partial n_{x}}\right|_{\partial \Omega}=0
\end{gathered}
$$

где $x \in \Omega, t>0$. При этом мы рассмотрим два вида области $\Omega$ : либо $\Omega \subset \mathbb{R}^{N}$ является ограниченной областью с гладкой границей, либо $\Omega \subset \mathbb{R}^{N}$ неограничена, $\Omega=\mathbb{R}^{N} \backslash \Omega_{0}$, где $\Omega_{0}$ - ограниченная область с гладкой границей $\partial \Omega_{0}$, содержащей начало координат.

Для исследования вопроса о достаточных условиях разрушения решения задачи (1.5), (1.6) воспользуемся методом нелинейной емкости Похожаева-Митидиери [2]. В работе [3] этим методом была исследована задача Коши и начально-краевая задача для широко известного уравнения Курамото-Сивашинского

$$
u_{t}+\Delta^{2} u+\Delta u=|\nabla u|^{2}
$$

Принципиальное отличие уравнения (1.7) от уравнения (1.5) состоит в том, что вместо нелинейности $|\nabla u|^{2}$ мы рассматриваем более сложную нелинейность $\left(|\nabla u|^{2}\right)_{t}$. В классической трактовке подхода нелинейной емкости, изложенного в работе [2], сложно доказать отсутствие глобального решения в слабом смысле, поскольку имеется слагаемое $u_{t t}$, которое после умножения на пробную функцию $\phi(x, t)$ дает в силу неравенства Гёльдера неподходящую оценку. Поэтому мы пользуемся подходом работы [3], согласно которому искомое дифференциальное неравенство по времени получается умножением на пробную функцию $\phi(x)$, порождающую функцию $\psi(x)$ как решение следующей краевой задачи в шаре $B_{R} \equiv\left\{x \in \mathbb{R}^{N}:|x|<R\right\}$ с выколотой точкой $x=0$ :

$$
\frac{d^{2} \psi(r)}{d r^{2}}+\frac{N-1}{r} \frac{d \psi(r)}{d r}=\phi(r), \quad 0<r<R,\left.\quad \frac{d \psi(r)}{\partial r}\right|_{r=R}=\left.\psi(r)\right|_{r=R}=0 .
$$

В соответствии с работой [3] введем обозначения

$$
B_{\rho_{0}} \equiv\left\{x \in \mathbb{R}^{N}:|x|<\rho_{0}\right\}, \quad \rho_{0}=\min _{x \in \partial \Omega_{0}}|x|, \quad B_{\rho_{0}} \subset \Omega_{0} .
$$

Выберем радиус $R>0$ настолько большим, что выполнено включение $\Omega_{0} \subset B_{R / 2}$. В работе [3] получена оценка

$$
\int_{B_{R} \backslash \Omega_{0}} \phi(x)\left|\nabla_{x} u(x, t)\right|^{2} d x \geqslant c(R, \phi)\left(\int_{B_{R} \backslash \Omega_{0}} \phi(x) u(x, t) d x\right)^{2},
$$

где

$$
c(R, \phi) \equiv \int_{B_{R} \backslash \Omega_{0}} \frac{|\nabla \psi(x)|^{2}}{\phi(x)} d x \leqslant \int_{\rho_{0} \leqslant|x| \leqslant R} \frac{|\nabla \psi(x)|^{2}}{\phi(x)} d x<+\infty .
$$


При этом существуют пробные функции $\phi(r)$ такие, что последний интеграл сходится. Далее остается проинтегрировать полученное дифференциальное неравенство первого порядка по времени. Отсюда получаются достаточные условия отсутствия глобального по времени решения.

Сложность применения этой техники к уравнению (1.5) заключается в том, что нелинейность имеет вид $\left(|\nabla u|^{2}\right)_{t}$. Именно этой проблеме посвящена настоящая работа.

\section{2. СИЛЬНАЯ ОБОБЩЕННАЯ ПОСТАНОВКА ЗАДАЧИ И ВЫВОД ДИФФЕРЕНЦИАЛЬНОГО НЕРАВЕНСТВА}

Дадим определение сильного обобщенного решения задачи (1.5).

ОПРЕДЕЛЕНИЕ 1. Функцию $u(x, t) \in \mathbb{C}^{(2)}\left([0, T] ; H^{1}(\Omega)\right)$, удовлетворяющую интегральному равенству

$$
\begin{gathered}
\int_{\Omega}\left[u_{t t}(x, t) \phi(x)+(\nabla \Delta \phi(x), \nabla u)-\left(|\nabla u|^{2}\right)_{t} \phi(x)\right] d x=0, \\
u(x, 0)=u_{0}(x) \in H^{1}(\Omega), \quad u^{\prime}(x, 0)=u_{1}(x) \in H^{1}(\Omega),
\end{gathered}
$$

для всех $\phi(x) \in \mathbb{C}^{\infty}(\Omega)$ таких, что $\partial_{x_{k}} \phi(x) \in \mathbb{C}_{0}^{\infty}\left(B_{R} \cap \Omega\right)$ при $k=\overline{1, N}$ (по условию $\left.\Omega_{0} \subset B_{R / 2}\right)$, и любых $t \in[0, T]$ при некотором $T>0$, назовем сильным обобщенным решением задачи (1.5), (1.6).

Сделаем общее предположение, которого будем придерживаться в этом и следующем разделах. Пусть существует такой момент времени $T>0$, что сильное обобщенное решение $u(x, t) \in \mathbb{C}^{(2)}\left([0, T] ; H^{1}(\Omega)\right)$ существует.

Рассмотрим сначала более сложный случай, когда $\Omega=\mathbb{R}^{N} \backslash \Omega_{0}$. Введем следующие функционалы:

$$
J(t) \equiv \int_{\Omega} \phi(x) u(x, t) d x, \quad I(t) \equiv \int_{\Omega} \phi(x)|\nabla u|^{2} d x, \quad \phi(x) \in \mathbb{C}^{\infty}(\Omega) .
$$

В качестве пробной функции $\phi(x)$ возьмем $\phi(|x|)=\phi(r) \geqslant 0$ вида

$$
\phi(r)= \begin{cases}1, & \text { если } 0 \leqslant r \leqslant R / 2, \\ 0, & \text { если } r \geqslant R .\end{cases}
$$

Справедлива следующая оценка:

$$
\begin{gathered}
\left|\int_{\Omega}(\nabla u(x, t), \nabla \Delta \phi(x)) d x\right| \leqslant \\
\leqslant\left(\int_{\Omega} \phi(x)|\nabla u|^{2} d x\right)^{1 / 2}\left(\int_{\Omega} \frac{|\nabla \Delta \phi|^{2}}{\phi} d x\right)^{1 / 2} \leqslant I(t)+c_{1}(\phi), \\
c_{1}(\phi) \equiv \frac{1}{4} \int_{\Omega} \frac{|\nabla \Delta \phi|^{2}}{\phi} d x .
\end{gathered}
$$

С учетом интегрального равенства в (2.1), оценки (2.4) и обозначений (2.2) получим дифференциальное неравенство второго порядка

$$
\frac{d^{2} J(t)}{d t^{2}}+c_{1}+I(t) \geqslant \frac{d I(t)}{d t}, \quad t>0 .
$$




\section{3. ИССЛЕДОВАНИЕ ДИФФЕРЕНЦИАЛЬНОГО НЕРАВЕНСТВА}

В этом разделе мы исследуем дифференциальное неравенство (2.5). Очевидно, его можно переписать в следующем виде:

$$
e^{-t} \frac{d^{2} J(t)}{d t^{2}} \geqslant \frac{d}{d t}\left(e^{-t} I(t)\right)-c_{1} e^{-t} .
$$

Справедливо следующее равенство:

$$
\int_{0}^{t} e^{-s} J^{\prime \prime}(s) d s=\left.e^{-s} J^{\prime}(s)\right|_{s=0} ^{s=t}+\left.e^{-s} J(s)\right|_{s=0} ^{s=t}+\int_{0}^{t} J(s) e^{-s} d s .
$$

С учетом этого равенства и начальных условий $J_{0}=J(0), J_{1}=J^{\prime}(0)$ и $I_{0}=I(0)$ из неравенства (3.1) интегрированием по времени приходим к дифференциальному неравенству первого порядка

$$
e^{-t} \frac{d J(t)}{d t}+e^{-t} J(t)+\int_{0}^{t} e^{-s} J(s) d s-J_{1}-J_{0} \geqslant e^{-t} I(t)-I_{0}-c_{1}\left(1-e^{-t}\right) .
$$

Теперь воспользуемся результатом работы [3] и получим следующую оценку функционала $I(t)$ снизу:

$$
I(t) \geqslant k J^{2}(t), \quad k \equiv\left[\int_{\Omega} \frac{|\nabla \psi(x)|^{2}}{\phi(x)} d x\right]^{-1},
$$

где функция $\psi(x)$ является решением краевой задачи (1.8). Таким образом, из неравенства (3.3) мы получаем неравенство

$$
\frac{d J(t)}{d t}+J(t)+\int_{0}^{t} e^{t-s} J(s) d s \geqslant k J^{2}(t)+\left(J_{1}+J_{0}-I_{0}-c_{1}\right) e^{t}+c_{1},
$$

которое можно переписать как

$$
e^{-t} \frac{d}{d t}\left(e^{t} J(t)\right)+\int_{0}^{t} e^{t-s} J(s) d s \geqslant k J^{2}(t)+E_{0} e^{t}+c_{1}, \quad E_{0}=J_{1}+J_{0}-I_{0}-c_{1} .
$$

Введем обозначение

$$
\Phi(t) \equiv e^{t} J(t) .
$$

С учетом этого обозначения из (3.6) получаем неравенство

$$
\frac{d \Phi(t)}{d t}+\int_{0}^{t} e^{2(t-s)} \Phi(s) d s \geqslant k e^{-t} \Phi^{2}(t)+E_{0} e^{2 t}+c_{1} e^{t} .
$$

Предположим, что

$$
\Phi(0)=J_{0} \geqslant 1, \quad J_{1}+J_{0} \geqslant I_{0}+\left.c_{1} \quad \Longrightarrow \quad \frac{d \Phi(t)}{d t}\right|_{t=0}>0 .
$$

Поскольку $u(x, t) \in \mathbb{C}^{(2)}\left([0, T] ; H^{1}(\Omega)\right)$ при некотором $T>0$, найдется такое $t_{1}>0$, что

$$
\frac{d \Phi(t)}{d t}>0 \quad \text { при } \quad t \in\left[0, t_{1}\right] .
$$


Следовательно, $\Phi\left(s_{1}\right) \geqslant \Phi\left(s_{2}\right)$ при $s_{1} \geqslant s_{2}, s_{1}, s_{2} \in\left[0, t_{1}\right]$, и поэтому из неравенства (3.8) мы получаем неравенство

$$
\frac{d \Phi(t)}{d t}+\frac{1}{2}\left(e^{2 t}-1\right) \Phi(t) \geqslant k e^{-t} \Phi^{2}(t)+E_{0} e^{2 t}+c_{1} e^{t}
$$

Введем следующие обозначения:

$$
h_{1}(t) \equiv \frac{e^{2 t}-1}{2}, \quad h_{2}(t) \equiv \int_{0}^{t} h_{1}(s) d s=\frac{e^{2 t}-1-2 t}{4} .
$$

С учетом этих обозначений из (3.11) мы получаем неравенство

$$
e^{-h_{2}(t)} \frac{d}{d t}\left(e^{h_{2}(t)} \Phi(t)\right) \geqslant k e^{-t} \Phi^{2}(t)+E_{0} e^{2 t}+c_{1} e^{t}
$$

и, положив

$$
\Psi(t) \equiv e^{h_{2}(t)} \Phi(t)
$$

приходим к следующему неравенству:

$$
\frac{d \Psi(t)}{d t} \geqslant k e^{-t-h_{2}(t)} \Psi^{2}(t)+E_{0} e^{2 t+h_{2}(t)}+c_{1} e^{t+h_{2}(t)} \geqslant k e^{-h_{3}(t)} \Psi^{2}(t),
$$

где

$$
h_{3}(t)=t+h_{2}(t)=\frac{2 t+e^{2 t}-1}{4} .
$$

Решая неравенство (3.15), получаем, что

$$
J(t) \geqslant \frac{J_{0} e^{-h_{3}(t)}}{1-k J_{0} \int_{0}^{t} e^{-h_{3}(s)} d s}
$$

Итак, мы вывели основное условие отсутствия глобального сильного обобщенного решения на конечном временно́м интервале:

$$
k J(0) \int_{0}^{+\infty} e^{-h_{3}(t)} d t>1
$$

При этом возникают два случая: либо для всех $t \in[0, T]$ производная $\Phi^{\prime}(t)>0$, либо найдется такое $t_{1} \in(0, T)$, что $\Phi^{\prime}\left(t_{1}\right)=0$. При этом если

$$
k J(0) \int_{0}^{t_{1}} e^{-h_{3}(t)} d t=1
$$

то решение неравенства (3.17) разрушается за конечное время. В частности, функционал $J(t)$ не может быть определен для всех $t>0$. Поэтому предположим, что в некоторый момент времени $t_{1}$

$$
\Phi^{\prime}\left(t_{1}\right)=0, \quad k J(0) \int_{0}^{t_{1}} e^{-h_{3}(t)} d t<1 .
$$


Получим достаточные условия, при которых последнее неравенство не выполнено. Из условия $\Phi^{\prime}\left(t_{1}\right)=0$ и из неравенства (3.8) получаем

$$
\Phi\left(t_{1}\right) \frac{e^{2 t_{1}}-1}{2} \geqslant \int_{0}^{t_{1}} e^{2\left(t_{1}-s\right)} \Phi(s) d s \geqslant k e^{-t_{1}} \Phi^{2}\left(t_{1}\right)+E_{0} e^{2 t_{1}}+c_{1} e^{t_{1}} \geqslant k e^{-t_{1}} \Phi^{2}\left(t_{1}\right) .
$$

Отсюда с учетом (3.9) мы приходим к неравенству $\Phi\left(t_{1}\right) \geqslant 1$ :

$$
\Phi^{2}\left(t_{1}\right) \frac{e^{2 t_{1}}-1}{2} \geqslant k e^{-t_{1}} \Phi^{2}\left(t_{1}\right) \quad \Longrightarrow \quad e^{3 t_{1}} \geqslant e^{t_{1}}+2 k \quad \Longrightarrow \quad e^{3 t_{1}} \geqslant 1+2 k .
$$

Теперь рассмотрим неравенство из (3.19). Заметим, что $h_{3}(t) \geqslant t$, поэтому

$$
k J_{0}\left[1-e^{-t_{1}}\right]<1 \quad \Longrightarrow \quad e^{t_{1}}<\frac{k J_{0}}{k J_{0}-1} .
$$

Используя неравенства (3.21) и (3.22), мы получаем

$$
\left(\frac{k J_{0}}{k J_{0}-1}\right)^{3}>1+2 k
$$

Отметим, что это неравенство не выполняется при достаточно большом $J_{0}>0$.

Таким образом, мы получили следующие достаточные условия отсутствия глобального сильного обобщенного решения начально-краевой задачи (1.5):

$$
\begin{gathered}
J_{0}>\max \left\{1, \frac{1}{k}, \frac{1}{k}\left[\int_{0}^{+\infty} e^{-h_{3}(t)} d t\right]^{-1}\right\}, \quad J_{1}+J_{0} \geqslant I_{0}+c_{1}, \\
\left(\frac{k J_{0}}{k J_{0}-1}\right)^{3} \leqslant 1+2 k, \quad k=\left[\int_{\mathbb{R}^{N}} \frac{|\nabla \psi(x)|^{2}}{\phi(x)} d x\right]^{-1} .
\end{gathered}
$$

Заметим, что в силу оценки (3.4) и определения (2.2) функционалов $I(t)$ и $J(t)$ выполнены неравенства

$$
\frac{1}{k^{1 / 2}}\left(\int_{\Omega}|\nabla u|^{2} d x\right)^{1 / 2} \geqslant \frac{1}{k^{1 / 2}} I^{1 / 2}(t) \geqslant J(t) .
$$

Справедлива следующая теорема.

Теорема 1. Предположим, что существует $T>0$, при котором определено сильное обобщенное решение начально-краевой задачи (1.5), (1.6) и выполнены условия (3.24), (3.25). Тогда имеет место неравенство

$$
J(t) \geqslant \frac{J_{0} e^{-h_{3}(t)}}{1-k J_{0} \int_{0}^{t} e^{-h_{3}(s)} d s} \quad \text { npu } \quad t \in[0, T], \quad T<t_{1},
$$

где момент времени $t_{1}>0$ определяется условием

$$
k J_{0} \int_{0}^{t_{1}} e^{-h_{3}(s)} d s=1, \quad h_{3}(t)=\frac{2 t+e^{2 t}-1}{4} .
$$

Таким образом, при указанных условиях силъное обобщенное решение не существует глобально во времени. 


\section{4. НАЧАЛЬНО-КРАЕВАЯ ЗАДАЧА (1.5), (1.6) В ОГРАНИЧЕННОЙ ОБЛАСТИ}

Рассмотрим теперь начально-краевую задачу в ограниченной области $\Omega \subset \mathbb{R}^{N}$ :

$$
\begin{gathered}
u_{t t}-\Delta^{2} u=\left(|\nabla u|^{2}\right)_{t}, \quad(x, t) \in Q_{T} \equiv \Omega \times(0, T], \\
u=\frac{\partial}{\partial n} \Delta u=0 \quad \text { на } \quad \Gamma_{T} \equiv \partial \Omega \times(0, T), \\
u(x, 0)=u_{0}(x), \quad u^{\prime}(x, 0)=u_{1}(x), \quad x \in \bar{\Omega} .
\end{gathered}
$$

Эта задача аналогична задаче для уравнения Курамото-Сивашинского, рассмотренной в работе [3]. Естественно, при соответствующем определении слабого решения справедлива теорема 1, поскольку при указанных граничных условиях (4.2) мы получаем то же самое дифференциальное неравенство (2.5). Но результат о разрушении сильного обобщенного решения задачи (4.1)-(4.3) можно получить более очевидным образом.

Дадим определение сильного обобщенного решения задачи (4.1)-(4.3).

ОПРЕДЕЛЕНИЕ 2. Силъным обобщенным решением задачи (4.1)-(4.3) назовем функцию $u(x, t) \in \mathbb{C}^{(2)}([0, T] ; \mathbb{W})$, где

$$
\mathbb{W} \equiv\left\{v(x) \in \mathbb{W}^{2,4}(\Omega),\left.v\right|_{\partial \Omega}=\left.\frac{\partial \Delta v}{\partial n}\right|_{\partial \Omega}=0\right\}
$$

удовлетворяющую интегральному равенству

$$
\int_{\Omega}\left[u_{t t}-\Delta^{2} u-\left(|\nabla u|^{2}\right)_{t}\right] \phi(x) d x=0, \quad t \in[0, T], \quad T>0,
$$

для всех $\phi(x) \in \mathbb{C}(\bar{\Omega})$, а также начальным условиям

$$
u(x, 0)=u_{0}(x) \in \mathbb{W}^{2,4}(\Omega), \quad u^{\prime}(x, 0)=u_{1}(x) \in \mathbb{W}^{2,4}(\Omega) .
$$

Введем следующие обозначения:

$$
I_{1}(t) \equiv \int_{\Omega} u(x, t) d x, \quad J_{1}(t) \equiv \int_{\Omega}|\nabla u|^{2} d x .
$$

Возьмем в интегральном равенстве (4.4) в качестве пробной функции $\phi(x)=1$ и тогда с учетом обозначений (4.5) в силу второго граничного условия (4.2) получим следующее равенство:

$$
\frac{d^{2} I_{1}(t)}{d t^{2}}=\frac{d J_{1}(t)}{d t} .
$$

Интегрируя его по времени, имеем

$$
\frac{d I_{1}(t)}{d t}=J_{1}(t)+I_{11}-J_{10}, \quad I_{11} \equiv I_{1}^{\prime}(0), \quad J_{10} \equiv J_{1}(0) .
$$

В силу неравенства Фридрихса справедливы цепочки оценок снизу

$$
J_{1}(t) \geqslant \lambda_{1} \int_{\Omega}|u|^{2} d x \geqslant k_{1}^{2} I_{1}^{2}(t), \quad k_{1} \equiv \lambda_{1}^{1 / 2}|\Omega|^{-1},
$$


где $\lambda_{1}>0$ - это первое собственное значение первой краевой задачи на собственные функции и собственные значения для оператора Лапласа в ограниченной области $\Omega$.

С учетом неравенства (4.8) из дифференциального равенства (4.7) получаем искомое дифференциальное неравенство

$$
\frac{d I_{1}(t)}{d t} \geqslant k_{1}^{2} I_{1}^{2}(t)+c_{2}, \quad c_{2} \equiv I_{11}-J_{10}=\int_{\Omega}\left[u_{1}(x)-\left|\nabla u_{0}(x)\right|^{2}\right] d x .
$$

Это дифференциальное неравенство легко интегрируется (см., например, работу [4]), и из него вытекают достаточные условия разрушения сильного обобщенного решения задачи (4.1)-(4.3) за конечное время. Справедлива следующая теорема.

ТЕОРема 2. Пусть существует $T>0$, при котором определено сильное обобщенное решение задач (4.1)-(4.3) в смысле определения 2, тогда справедливы следующие свойства.

1. Если $c_{2}=a^{2}>0$, то имеют место неравенства

$$
I_{1}(t) \geqslant \frac{a}{k_{1}} \operatorname{tg}\left(a k_{1} t+c_{3}\right), \quad T<\frac{\pi / 2-c_{3}}{a k_{1}}, \quad c_{3}=\operatorname{arctg} \frac{k I_{10}}{a} .
$$

2. Если $c_{2}=0$, то имеют место неравенства

$$
I_{1}(t) \geqslant \frac{I_{10}}{1-I_{10} k_{1}^{2} t}, \quad T<\frac{1}{k_{1}^{2} I_{10}} .
$$

3. Если $c_{2}=-a^{2} \neq 0$, то имеют место неравенства

$$
I_{1}(t) \geqslant \frac{1+c_{4} e^{2 a k_{1} t}}{1-c_{4} e^{2 a k_{1} t}} \frac{a}{k_{1}}, \quad T<\frac{1}{2 a k} \ln \frac{k I_{10}+a}{k I_{10}-a}, \quad c_{4}=\frac{k_{1} I_{10}-a}{k I_{10}+a},
$$

где $I_{10}=I_{1}(0)$.

ЗАМЕЧАНИЕ 1. В силу очевидного вложения $\mathbb{W} \subset \mathbb{W}_{0}^{2,1}(\Omega)$ и неравенства (4.8) мы приходим к выводу о том, что если выполнены (достаточные) условия теоремы 2, то не существует глобальное во времени сильное обобщенное решение задачи (4.1)-(4.3).

ЗАмЕчАниЕ 2. Уравнения вида (1.1) с характерной нелинейностью $\left(|\nabla u|^{2}\right)_{t}$ систематически возникают в нелинейной физике и составляют неисследованный класс нелинейных уравнений математической физики, представляющий значительный интерес. Мы полагаем, что представленная работа является первой в серии исследований таких нелинейных уравнений.

\section{Список литературы}

[1] Э. Инфельд, Дж. Роуландс, Нелинейные волны, солитоны и хаос, М., Физматлит, 2006.

[2] Э. Митидиери, С. И. Похожаев, Тр. МИАН, 234, 2001, 3-383.

[3] С. И. Похожаев, Матем. сб., 199:9 (2008), 97-106.

[4] V.A. Galaktionov, E. Mitidieri, S. I. Pohozaev, Nonlinear Anal., 70:8 (2009), 2930-2952. 